\title{
Erratum to: Non-self-averaging of a two-person game with only positive spillover: a new formulation of Avatamsaka's dilemma
}

\author{
Yuji Aruka • Eizo Akiyama
}

Published online: 6 March 2010

(C) Springer-Verlag 2010

\section{Erratum to: J Econ Interact Coord (2009) 4:135-161} DOI 10.1007/s11403-009-0050-1

On page 141, the first line of Prisoner's dilemma reads: 1 should be replaced by 0.7 . On page 141 , the first element of Table 2 reads: $(1,1)$ should be replaced $(0.7,0.7)$.

The online version of the original article can be found under doi:10.1007/s11403-009-0050-1.

Y. Aruka $(\varangle)$

Faculty of Commerce, Chuo University,

742-1 Higashinakano, Hachioji, Tokyo 192-0393, Japan

e-mail: aruka@tamacc.chuo-u.ac.jp

E. Akiyama

Graduate School of Systems and Information Engineering, University of Tsukuba,

Tennodai 1-1-1, Tsukuba, Ibaraki 305-8573, Japan

e-mail: eizo@sk.tsukuba.ac.jp 